\title{
Formações venosas superficiais da fossa cubital: aspectos de interesse para a prática da Enfermagem
}

\author{
Superficial venous formation of the cubital fossa: aspects of interest for nursing practice
}

Formaciones venosas superficiales de la fosa cubital: aspectos de interés para la práctica de Enfermería

\begin{abstract}
Nilton Alves'
' Universidade Estadual Paulista, Departamento de Morfologia. Araraquara-SP, Brasil. Universidad de Talca, Facultad de Ciencias de la Salud, Departamento de Ciencias Biomedicas (Professor Visitante). Talca, Chile.
\end{abstract}

Submissão: 19-04-2011 Aprovação: 20-12-2012

\begin{abstract}
RESUMO
O objetivo deste estudo é contribuir para o conhecimento que auxilie o profissional de enfermagem na identificação dos tipos mais comuns de formações venosas da região da fossa cubital e, ainda, enfocar a importância de estar sempre atento aos casos pouco comuns como o aqui relatado. Através de uma revisão bibliográfica, constatamos que as formações venosas dessa região podem ser classificadas em 5 tipos mais comuns, sendo o tipo II o mais frequente. Constatamos ainda, que a VICo é o local de punção mais indicado, seguido pela VIB. Descrevemos também uma variação anatômica, onde observamos ausência de comunicação entre VC e VB no nível da fossa cubital e VIA drenando na VB, estando presente a VCA.
\end{abstract}

Descritores: Anatomia; Antebraço; Veias; Enfermagem.

\begin{abstract}
The aim of this study is to contribute to the knowledge to assists the nursing staff to identify the most common types of venous formations of the cubital fossa region, and also focus on the importance of always being alert to unusual cases as that reported here. Through a literature review, we found that the venous formations of this region can be classified into five common types, bring the Type II the was most frequent. We also found that MCV is considered the best puncture site, followed by MBV. We also describe an anatomical variation, in which we observed the absence of communication between BV and CV at the level of cubital fossa draining into BV and MVF, with the presence of the ACV.
\end{abstract}

Key words: Anatomy; Forearm; Venous; Nursing.

\section{RESUMEN}

El objetivo de esta investigación es contribuir al conocimiento que auxilie al profesional de enfermería en la identificación de los tipos más comunes de formaciones venosas de la fosa cubital, además de advertir sobre la importancia de fijar la atención a los casos poco comunes, como lo aquí reportado. A través de la revisión bibliográfica, clasificamos las formaciones venosas de esta región en cinco tipos más comunes, siendo lo más frecuente el Tipo II. La utilización de la VICo se recomienda como el mejor sitio de punción, seguido por la VIB. Además, describimos una variación anatómica, donde se observó la ausencia de comunicación entre VC y VB a nivel de fosa cubital y VIA drenando en VB, con presencia de la VCA.

Palabras clave: Anatomía; Antebrazo; Venas; Enfermería. 


\section{INTRODUÇÃO}

$\mathrm{Na}$ fossa cubital encontram-se veias de grande calibre, onde são realizados inúmeros procedimentos médicos, sendo um dos locais mais importantes para a realização de punções venosas. Nessa região são realizadas injeções endovenosas, administração de soluções parenterais, plasma e medicamentos $^{(1-2)}$, introdução de cateteres para obtenção de sangue a partir das câmaras cardíacas e cardioangiografia ${ }^{(1-3)}$.

A realização de punções venosas periféricas representa em torno de $85 \%$ das atividades executadas pelos profissionais enfermagem ${ }^{(4-5)}$, embora seja realizada nas veias superficiais, a punção venosa é um procedimento invasivo ${ }^{(6)}$. Geralmente, é guiado pela inspeção da anatomia das veias periféricas do paciente, exigindo conhecimento, treinamento específico e habilidade, uma vez que muitos fatores podem interferir na identificação da rede venosa ${ }^{(7)}$.

Num estudo em recém-nascidos, alguns autores assinalaram que $64,42 \%$ das vias eram instaladas em uma primeira punção e $35,58 \%$ necessitam mais de uma tentativa ${ }^{(8)}$. Em outro estudo em recém-nascidos, os autores constataram que existe desconhecimento por parte dos profissionais de enfermagem na eleição das veias em intervenções que exijam acesso venoso ${ }^{(9)}$.

A falta de conhecimento científico e técnico pode determinar a execução incorreta deste procedimento, podendo causar ao paciente dor leve local, dor crônica, infecções, novas e/ou múltiplas punções, infiltrações, hemorragia subcutânea e atraso na terapêutica ${ }^{(10)}$. Além disso, pode ocorrer comprometimento nervoso, causando a chamada síndrome da dor regional complexa, uma vez que os nervos cutâneo medial e lateral do antebraço emitem ramos que se apresentam bastante superficiais na região cubital $\left.\right|^{(1,6,11-12)}$.

As veias superficiais do membro superior constam basicamente de duas veias de grande calibre, as veias cefálica e basílica, claramente visíveis quando se aplica um torniquete no braço. Elas se originam da rede venosa dorsal da mão, sendo que a veia cefálica $(\mathrm{VC})$ ascende lateralmente na face anterior do antebraço enquanto que a veia basílica (VB) ascende medialmente na face anterior do antebraço. Em seu trajeto, estas veias recebem diversas tributárias, mantendo a comunicação entre si ${ }^{(2,13)}$. Destas comunicações devemos destacar a veia intermédia do cotovelo (VICo) que comumente apresenta-se com um trajeto oblíquo dirigindo-se mais para proximal a partir da VC até a VB recebendo a veia intermédia do antebraço (VIA) como tributária(1-2).

Não é possível estabelecer um padrão rígido em relação à distribuição venosa na fossa cubital, uma vez que as variações anatômicas são muito frequentes.

\section{OBJETIVO}

O objetivo deste trabalho é contribuir para o conhecimento que facilite a identificação dos tipos mais comuns de formações venosas da região da fossa cubital, e relatar uma variação anatômica pouco comum, procurando, assim, auxiliar na prática diária dos procedimentos de enfermagem.

\section{MÉTODOS}

Foi realizado um levantamento bibliográfico a fim de relatar os tipos de formações venosas descritos na literatura. Neste estudo foram incluídos artigos científicos e livros editados nas línguas inglesa, espanhola, portuguesa e francesa, no período de 1908 a 2010. Foram selecionados artigos publicados nas bases ISI, Scielo, Lilacs, Medline, Science Direct (Elselvier), SpringerLink Books (Metapress), Willey Interscience Journals. Foram realizados leitura, análise e registro dos dados obtidos.

Para a classificação das formações venosas foi escolhida a classificação proposta por Del Sol et al.(3), sendo a mesma adaptada para este estudo (Quadro 1), conforme descrição abaixo:

Tipo I: Veia Cefálica (VC) se divide em Veia Intermédia Basílica (VIB) e Veia Intermédia Cefálica (VIC) unindo-se à Veia Basílica (VB) e Veia Cefálica Acessória (VCA), respectivamente.

Tipo II: VC origina a Veia Intermédia do Cotovelo (VICo) que se une à VB. Não existe VCA e a VIA (Veia intermédia do antebraço) drena em VB.

Tipo III: não existe comunicação entre VB e VC no nível da fossa cubital. A VIA drena na VB.

Tipo IV: VC drena em VB e a VIA drena na VC.

Tipo V: M clássica e outras disposições.

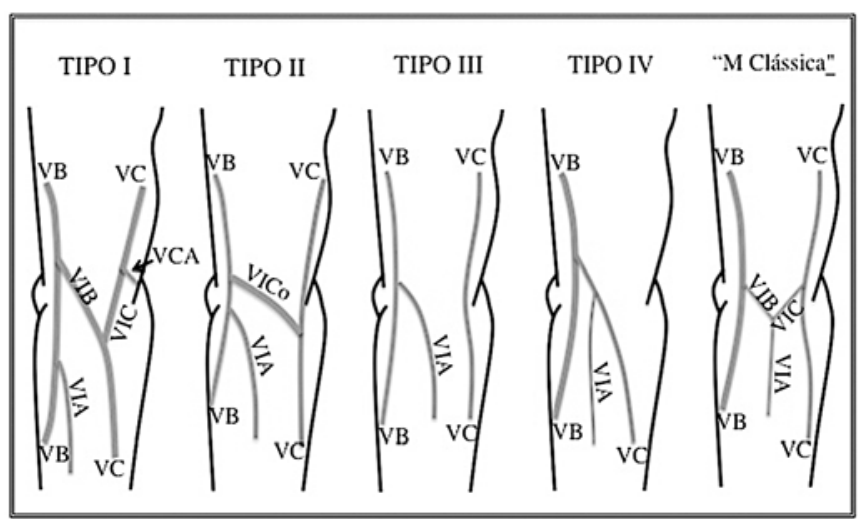

Legenda: VC - Veia Cefálica; VB - Veia Basílica; VIA - Veia Intermédia do Antebraço; VCA - Veia Cefálica Acessória; VIC - Veia Intermédia Cefálica; VIB - Veia Intermédia Basílica; VICo - Veia Intermédia do Cotovelo.

Quadro 1 - Principais tipos de formações venosas da região de fossa cubital. Esquema representando membro superior esquerdo.

Além da análise dos artigos científicos, descreve-se também um caso incomum de formação venosa.

\section{Apresentação do Caso}

O presente trabalho descreve uma variação anatômica encontrada no membro superior esquerdo de um cadáver adulto do sexo masculino pertencente ao laboratório de Anatomia da Universidade de Talca - UTALCA, Chile. Foi observado que não existia comunicação entre VC e VB no nível da fossa cubital e a VIA drenava na VB. Além disso, observamos a presença da VCA (Figura 1). 


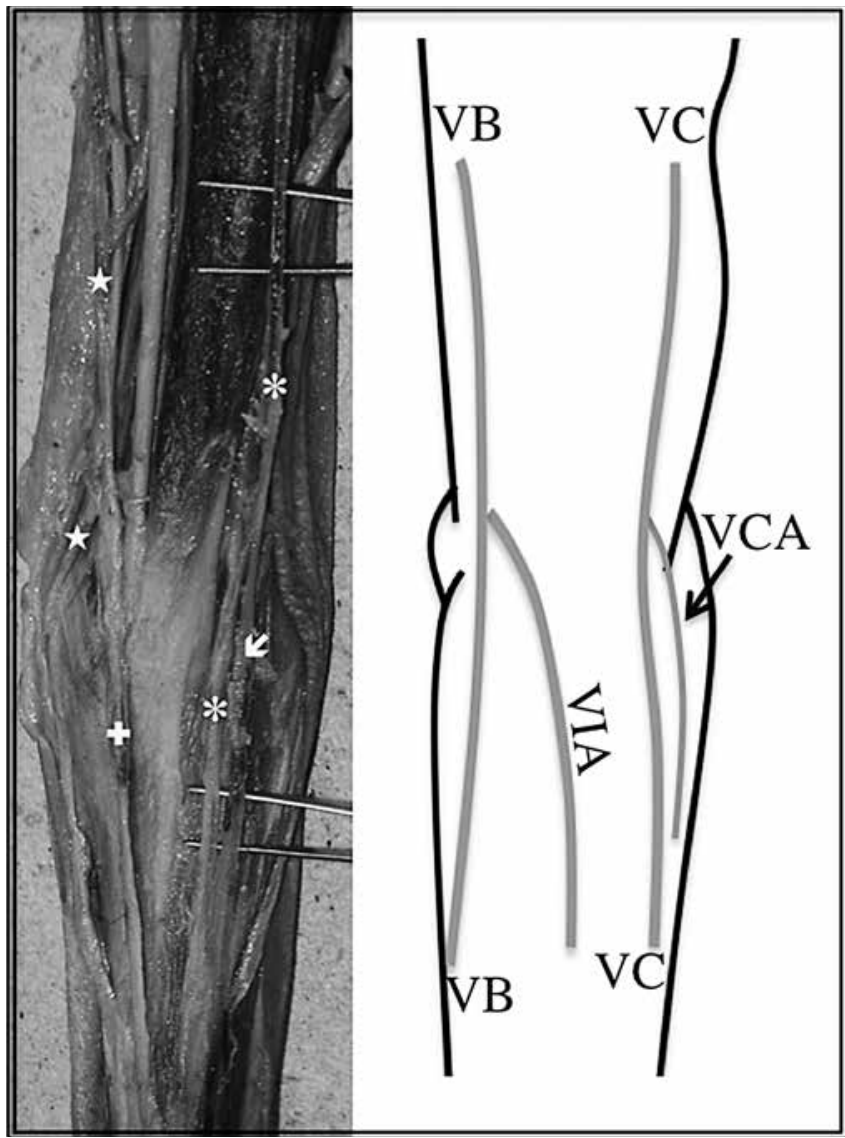

Figura 1 - Foto de membro superior esquerdo apresentando variação anatômica com esquema demonstrativo. VB - Veia Basílica (|); VC - Veia Cefálica $\left(^{*}\right)$; VIA - Veia Intermédia do Antebraço $(\rightarrow)$; VCA Veia Cefálica Acessória (í)

\section{RESULTADOS E DISCUSSÃO}

No quadro 2 apresentamos os tipos de formações venosas e as porcentagens destas segundo literatura científica.

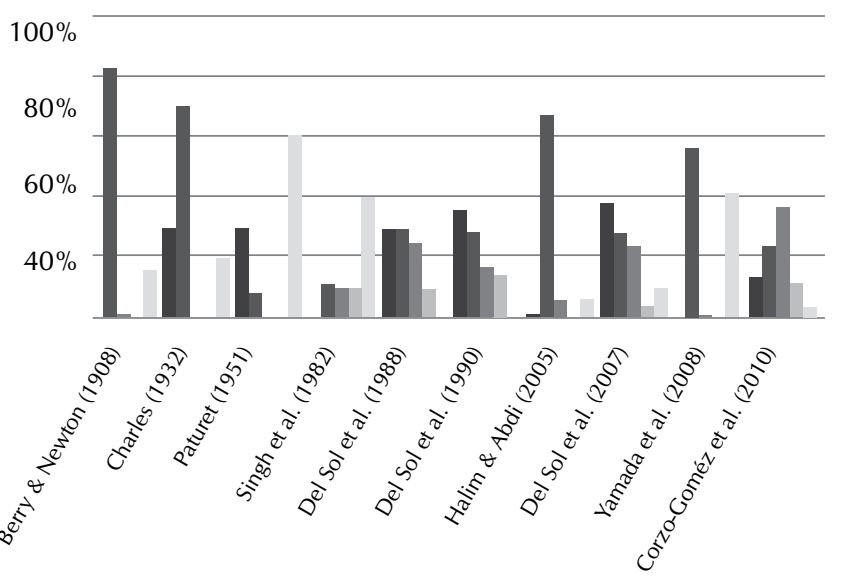

口TIPO I —TIPO II —TIPO III TIPO IV TIPO V

Quadro 2 - Frequência (\%) dos tipos de formações venosas relatadas em literatura.
As veias superficiais da fossa cubital apresentam grande número de variações anatômicas; porém, é possível estabelecer os padrões mais comuns relatados pelos autores.

Para Del Sol et al. ${ }^{(3)}$ e Del Sol et al. ${ }^{(14)}$ o padrão encontrado com maior frequência foi o tipo I (Quadro 1), apresentando porcentagens de 38,7\% e 36,25\%, respectivamente. Charles ${ }^{(15)}$, Paturet ${ }^{(16)}$ e Del Sol et al. ${ }^{(17)}$ encontraram frequências bastante similares com $30 \%, 27,5 \%$, e $30 \%$, respectivamente. Corzo-Goméz et al. ${ }^{(18)}$ relatam que este padrão foi o terceiro mais encontrado em seu estudo, com 14\% dos casos (Quadro 2).

Com relação ao tipo II (Quadro 1), no qual se observa a VICo unindo a VC à VB na região de fossa cubital, Yamada et al. ${ }^{(6)}$, Charles ${ }^{(15)}$, Barry \& Newton ${ }^{(19)}$ e Halim \& Abdi ${ }^{(20)}$ relataram tratar-se de um padrão muito frequente, com 56,7\%, $70 \%, 83 \%$, e $67,5 \%$ dos casos, respectivamente. Gardner, Gray, \& O'rahilly ${ }^{(21)}$ também relataram tratar-se de uma formação bastante frequente. Outros autores encontraram procentagens menores, porém bastante expressivas, com 28,3\% para Del Sol et al. ${ }^{(3)}, 28,75 \%$ para Del Sol et al. ${ }^{(14)}, 30 \%$ para Del Sol et al. ${ }^{(17)}$ e $24 \%$ para Corzo-Goméz et al. ${ }^{(18)}$. Valores menos expressivos foram encontrados por Paturet ${ }^{(16)}$, com 8\% e Singh et al. ${ }^{(22)}$, com $11 \%$ (Quadro 2).

O Tipo III (Quadro 1), onde observamos a ausência de comunicação entre VB e VC no nível da fossa cubital, foi o tipo encontrado com maior frequência por Corzo-Goméz et al. ${ }^{(18)}$, com $37 \%$ dos casos. Del Sol et al. ${ }^{(3)}$ e Del Sol et al. ${ }^{(17)}$ relataram ter encontrado este padrão em $24 \%$ e $25 \%$ dos indivíduos pesquisados, respectivamente. Del Sol et al. ${ }^{(14)}$, Halim \& Abdi $^{(20)}$ e Singh et al. ${ }^{(22)}$, encontraram valores menores, sendo estes $17,25 \%, 6,0 \%$ e $10 \%$, respectivamente. Esse tipo de formação foi bastante incomum para Barry \& Newton ${ }^{(19)}$, com $1 \%$, sendo que Yamada et al. ${ }^{(6)}$ não a encontraram (Quadro 2).

O tipo IV (Quadro 1), formado pela VC drenando em VB e a VIA drenando na VC, apresentou-se em 14,75\% para Del Sol et al. ${ }^{(14)}, 10 \%$ dos casos para Del Sol et al. ${ }^{(17)}$ e Singh et al. ${ }^{(22)}, 12 \%$ para Corzo-Goméz et al. ${ }^{(18)}$ e $19,5 \%$ para Halim \& $\mathrm{Abdi}^{(20)}$. Cabe ressaltar que esta formação é pouco encontrada por outros autores ${ }^{(3,15)} \mathrm{e}$, segundo Okamoto ${ }^{(23)}$, não foi encontrada na população japonesa (Quadro 2).

Para Paturet ${ }^{(16)}$ e Singh et al. ${ }^{(22)} \mathrm{O}$ " $\mathrm{M}$ " clássico (Quadro 1), formado pela divisão da VIA em VIC e VIB no nível do cotovelo, representado pelo Tipo $\mathrm{V}$, foi o padrão encontrado com maior frequência; porém, para autores como Del Sol et al. ${ }^{(3,14,17)}$, Charles ${ }^{(15)}$ e Corzo-Goméz et al. ${ }^{(18)}$, essa é uma formação venosa encontrada com pouca frequência (Quadro 2).

Em nosso caso observamos que não existia comunicação entre as VC e VB na região da fossa cubital, e que a VIA drenava na VB, formação muito similar ao Tipo III, porém foi observada a presença da VCA, caracterizando um padrão de drenagem venosa pouco comum na fossa cubital (Quadro 1; Figura 1).

Para realizar a punção venosa em crianças de 0-1 anos alguns autores recomendam utilizar a VC ou a VIC seguida pela VIB e $\mathrm{VICo}^{(17)}$, enquanto outros recomendaram que em pacientes pediátricos deve-se utilizar VIB, VIC e VICo ${ }^{(24)}$.

Para a punção venosa em adultos, Gardner, Gray, \& $\mathrm{O}^{\prime}$ rahilly ${ }^{(21)}$ recomendaram utilizar a VICo ou uma de suas tributárias. Já Del Sol et al. ${ }^{(14)}$ recomendam utilizar a VIB ou VICo 
apesar de sua estreita relação com o nervo cutâneo medial do antebraço (NCMA) e a artéria braquial. Moore \& Dalley ${ }^{(1)}$ afirmam que, em geral, a VICo e a VB devem ser selecionadas para realizar os procedimentos. Del Sol et al. ${ }^{(3)}$ e Del Sol \& Olave $^{(25)}$ recomendam utilizar a VIC e VC quando tiverem um calibre semelhante à VIB ou VICo, a fim de minimizar os riscos de puncionar outras estruturas anatômicas importantes. Yamada et al. ${ }^{(6)}$ afirmam que o melhor lugar para uma punção é a VICo próximo às veias cutâneas radiais já que, em seu estudo, observaram que a VC e o NCMA eram adjacentes e tinham seu trajeto paralelo ao nervo cutâneo lateral do antebraço (NCLA). Além disso, observaram que a VB e a VIA eram paralelas e adjacentes ao NCMA. Del Sol \& Vásquez ${ }^{(26)}$ recomendam a utilização da VIB no Tipo I e da VICo no Tipo II.

\section{CONSIDERAÇÕES FINAIS}

Frente a estes dados não temos dúvidas em afirmar que é imprescindível que o profissional da saúde tenha conhecimento das diversas formações venosas e nervosas da região cubital e que além disso, deve fazer um estudo desta região antes de qualquer intervenção a fim de evitar os danos ocasionados ao paciente por um procedimento mal realizado.

Podemos afirmar ainda que, de acordo com a literatura consultada, a VICo é o local de punção mais indicado, seguido da VIB e que a disposição venosa mais frequente é a do Tipo II, seguida pelos Tipos I, V, III e IV. Deve-se, no entanto, considerar sempre as eventuais variações anatômicas que podem ocorrer, como a que foi descrita neste trabalho.

\section{REFERÊNCIAS}

1. Moore KL, Dalley AF. Anatomia orientada para a clínica. 4. ed. Rio de Janeiro: Guanabara Koogan; 2001.

2. Dangelo JG, Fattini CA. Anatomia Humana Sistêmica e Segmentar. 3. ed. Rio de Janeiro: Atheneu; 2007.

3. Del Sol M, Mardones LM, Bustos TE. Formaciones Venosas de la Fosa Cubital en el Individuo Mapuche. Estudio Bioscópico. Int J Morphol 2007;25(4):885-94.

4. Griffith HM, Thomas N, Griffith L. MDs bill for these routine nursing tasks. Am J Nurs 1991;91(1):22-7.

5. Phillips, LD. Manual de Terapia Intravenosa. 2. ed. Porto Alegre: Artmed; 2001.

6. Yamada K, Yamada K, Katsuda I, Hida T. Cubital fossa venipuncture sites based on anatomical variations and relationships of cutaneous veins and nerves. Clin Anat 2008;21(4):307-13.

7. Pereira RCC, Zanetti ML, Ribeiro KP. Tempo de permanência do dispositivo venoso periférico, in situ, relacionado ao cuidado de enfermagem, em pacientes hospitalizados. Med 2001;34:79-84.

8. Rivas-Lienqueo A, Rivas-Riveros E. Evaluación de protocolos de seguimiento de vías venosas periféricas. neonatología. Hospital Dr. Hernán Henríquez Aravena. mayo-junio de 2005. Cienc Enferme 2008;14(2):47-53.

9. Rodrigues ZS, Chaves EMC, Cardoso MVLML. Atuação do enfermeiro no cuidado com o cateter central de inserção periférica no recém-nascido. Rev Bras Enferm 2006;59(5):626-9.

10. Torres MM, Andrade D, dos Santos CB. Punción venosa periférica: evaluación de desempeño de los profesionales de enfermería. Rev Latino-Americana Enferm 2005;13(3):299-304.

11. Horowitz SH. Peripheral nerve injury and causalgia secondary to routine venipuncture. Neurol 1994;44: 962-4.

12. Rubin M, Safdieh JE. Netter: Neuroanatomia essencial. 4. ed. Rio de Jeniro: Elsevier; 2008.

13. Jacob S. Atlas de Anatomía Humana. Elsevier Science; 2003.
14. Del Sol M, Wuster A, Fritz I. Tipos de formaciones venosas en la fosa cubital en jóvenes chilenos. Rev Chil Tecnol Méd 1990;13(2):646-9.

15. Charles CM. On the arrangement of the superficial veins of the cubital fossa in American white and American negro males. Anat Rec 1932;54(1):9-14.

16. Paturet, G. Traite d'anatomie humaine. Paris: Masson; 1951. v.2.

17. Del Sol M, DeAngelis MA, Bolini PAD. Formações venosas na fossa cubital da criança. Pediatr Mod 1988; 23(4):225-31.

18. Corzo-Gómez EG, Gómez-Díaz OL, Niño-Mantilla ME, Rey Triana RJ, Pedraza Díaz LJ. Distribución de los Patrones Venosos de la Fosa Cubital en una Muestra de Personas Nacidas en Bucaramanga, Colombia. Int J Morphol 2010;28(4):1011-8.

19. Berry RJA, Newton HAS. A study of the superficial veins of the superior extremity in 300 living subjects. Anat Anz 1908;33(5):591-601.

20. Halim A, Abdi SHM. Superficial venous patterns in the cubital region of Indians. Anat Rec 1974;178(3): 631-6.

21. Gardner E, Gray DJ, O'rahilly RO. Anatomia: estudo regional do corpo humano. 4. ed. Rio de Janeiro: Guanabara Koogan; 1978.

22. Singh SP, Ekandem GJ, Bose ES. A study of the superficial veins of the cubital fossa in Nigerian subject. Acta Anat 1982;774:311-20.

23. Okamoto K. A study of the superficial veins in the superior extremity of live Japanese. Anat Rec 1922;23: 323-33.

24. Weinstein S, Plumer AL. Plumer's principles \& practice of intravenous therapy. 8. ed. Lippincott edition; 2007.

25. Del Sol M, Olave E. Venas de la fosa cubital en el hombre. Sitios de punción. Rev Chil Cs Méd Biol 1991; 1(1):49-53.

26. Del Sol M, Vásquez B. Venas Superficiales de la Fosa Cubital. Aspectos Anátomo-Clínicos y Antropológicos. Int J Morphol 2009;27(2):527-38. 